\title{
Exodontia em ovino santa inês: relato de
}

\section{caso}

\section{Tooth extraction in santa inês sheep: a case report}

\section{Resumo}

A saúde e o desempenho dos pequenos ruminantes estão diretamente ligados à qualidade de sua dentição. Em diversas regiões do mundo as enfermidades odontológicas são responsáveis por grandes perdas econômicas, decorrentes das dificuldades na preensão, mastigação dos alimentos e na ruminação. Este trabalho relata um caso de exodontia de primeiro molar em uma ovelha que apresentava emagrecimento progressivo, permitindo a conclusão de que quanto antes for realizado o diagnóstico, melhor será o prognóstico do paciente.

\section{Abstract}

Health and performance of small ruminants are directly linked to the quality of their dentition. Dental diseases are responsible for great economic losses in several regions in the world, due to difficulties in the grasping, chewing of food and rumination. The aim of this study was to report a case of first molar extraction in a sheep with progressive weight loss showing that the sooner the diagnosis is firmed, the better the patient's prognosis will be 
Joyce Cristina Ripi Fioruci ${ }^{1}$

Luana Venâncio Garcia ${ }^{1}$

Mariana de Andrade Ferreira ${ }^{1}$

Caio José Xavier Abimussi²

Allison Maldonado 3
Rodovia BR 153, km 338

Ourinhos /SP, Brasil

CEP: 19909-100

凶joycefioruci@gmail.com

\section{Palavras-chave}

Perdas econômicas. Exodontia. Produção.

Ovinos.

\section{Keywords}

Economic losses. Tooth extraction. Production. Sheep.

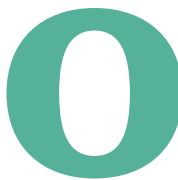

vinos e outros ruminantes possuem duas dentições sucessivas (difiodontes), com a dentição decídua composta por 20 dentes e a dentição permanente com 32. No lugar dos incisivos e caninos maxilares, os ruminantes possuem uma almofada dental, composta por tecido conjuntivo denso de colágeno, coberto por queratina (CUTRESS et al., 1972; WEINREB; SHARAV,1964). Nos ruminantes, cada grupo dentário assume papel independente. Os incisivos apresentam grande importância para a preensão dos alimentos e os dentes molares e pré-molares possuem papel primordial no processo mastigatório (PUGH, 2004). As doenças nos dentes e no periodonto afetam severamente o estado geral de saúde do animal (GREENE, 2001; PUGH, 2004). Em ruminantes, o diagnóstico de afecções orais é dificultado pelo não conhecimento de que tais doenças representam um problema sanitário de rebanhos; pelo pouco hábito dos profissionais da área; pelo fato dos criadores examinarem a cavidade oral dos animais; e pela falta de sintomatologia específica (WEINREB; SHARAV, 1964). O desgaste excessivo dos incisivos é comum em ovinos da Nova Zelândia e Austrália. Em algumas regiões, os dentes estão desgastados até o nível da gengiva antes dos três a quatro anos de idade (CHRISTIANSEN; KISSLING, 1986; KANE, 1984; ORR). Devido ao comprometimento da preensão dos alimentos, da mastigação e da ingestão de água, o animal fica

1 Discente do curso de Medicina Veterinária do Centro Universitário das Faculdades Integradas de Ourinhos (Unifio).

2 Docente do curso de Medicina Veterinária do Centro Universitário das Faculdades Integradas de Ourinhos (Unifio).

3 Departamento de Clínica e Cirurgia de Grandes Animais do Centro Universitário das Faculdades Integradas de Ourinhos (Unifio). 
vulnerável a infecções secundárias devido à dor e sensibilidade dentária ocasionadas pelo desgaste excessivo ou perda dental (SHERMAN, 1983).

$\mathrm{Na}$ Escócia, 70\% dos pequenos ruminantes eram descartados precocemente, mesmo antes do final da vida reprodutiva, por apresentarem problemas na dentição, gerando prejuízo financeiro de $30 \%$ a $40 \%$, pelo baixo escore corporal dos animais afetados (HERRTAGE; SAUNDERS; TERLECKI, 1974).

Este trabalho relata um caso de exodontia em um ovino santa inês de cinco anos de idade, descrevendo os aspectos clínicos, anestésicos e o tratamento cirúrgico do caso.

\section{Relato de caso}

No dia 4 de outubro de 2018, foi encaminhada para o setor de cirurgia de grandes animais do Hospital Veterinário Roque Quagliato do Centro Universitário das Faculdades Integradas de Ourinhos, uma ovelha da raça santa inês de cinco anos de idade, lactante, pesando $35 \mathrm{~kg}$, criada em regime intensivo, alimentada com feno, concentrado e sal mineral ad libitum.

Ao exame clínico o animal apresentava-se alerta, mucosa ocular levemente hipocorada e seu escore corporal analisado de acordo com a escala proposta por Jefferies (1961), que apresenta valores de 0 a 5, foi considerado como 1 . $\mathrm{O}$ animal apresentava emagrecimento progressivo e toda vez que se alimentava, parte do conteúdo caía de sua boca. $\mathrm{Na}$ inspeção da cavidade oral foi constatado que seus dentes apresentavam grandes falhas de oclusão, sendo esta imparcialidade palpada no exame físico pelo lado externo da face rostral.

No exame radiográfico foi verificada a presença de erupção dentária exacerbada do $1^{\circ}$ molar da maxila esquerda e diastema entre $4^{\circ}$ pré-molar e $1^{\circ}$ molar da mandíbula esquerda (Figura 1). Devido a este diastema entre os dentes da mandíbula, o $1^{\circ}$ molar estava crescendo exacerbadamente por causa da impossibilidade de ocorrer seu desgaste natural, sendo sugerido sua remoção cirúrgica.

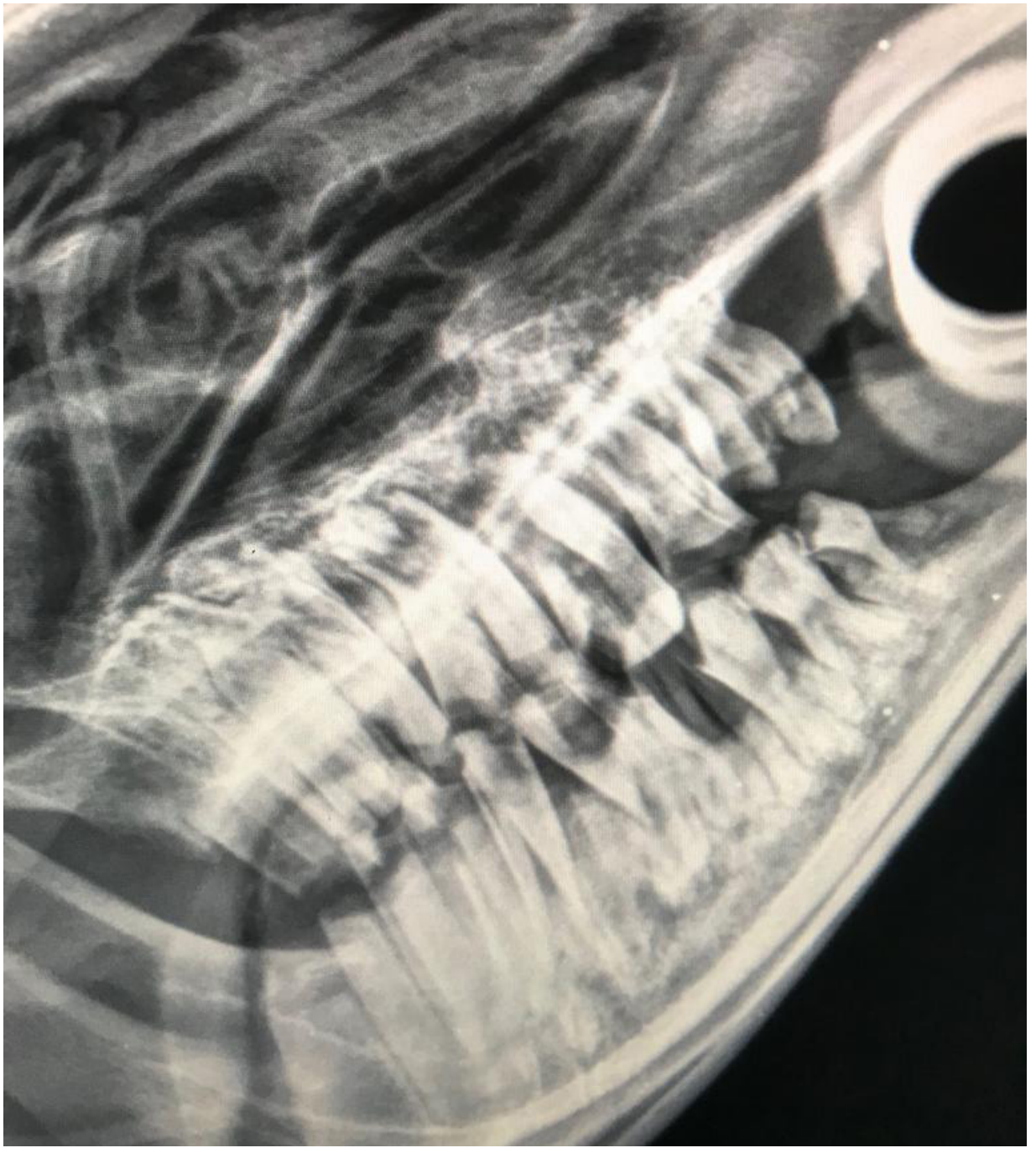

Figura 1 - Posicionamento látero-lateral de radiografia da arcada dentária. Fonte: Joyce C. R. Fioruci. 
No manejo pré-operatório, o animal foi submetido a jejum sólido e hídrico por 24 e 12 horas, respectivamente. Previamente ao procedimento cirúrgico, a boca foi higienizada com $500 \mathrm{ml}$ de uma solução composta por clorexidine alcoólico 5\%. Ato contínuo, foi administrado como medicação pré-anestésica $0,05 \mathrm{mg} / \mathrm{kg}$ de xilazina, $2 \%$ pela via intramuscular. Decorridos 15 minutos, um cateter $18 \mathrm{G}$ foi introduzido e fixado na veia cefálica esquerda, por onde foi administrado propofol na dose de $2,5 \mathrm{mg} / \mathrm{kg}$ associado com cetamina na dose de $1 \mathrm{mg} / \mathrm{kg}$.

Uma sonda orotraqueal, tamanho 10, foi introduzida pela cavidade oral até seu devido posicionamento na região da laringe e a acoplada a um circuito anestésico com reinalação de gases. Desta forma, o animal foi mantido sob anestesia inalatória com isofluorano, respeitando os estágios e planos de anestesia propostos por Guedel (Estágio III/Plano 2). Como anestesia local, foi realizado o bloqueio do nervo infraorbitário esquerdo com bupivacaína $0,5 \%$ sem vasoconstritor, na dose de $1 \mathrm{mg} / \mathrm{kg}$, utilizando uma agulha $25 \times 0,7$.

No centro cirúrgico o abre-boca foi colocado no animal e a desmotomia dos ligamentos periodontais foi realizada com o emprego do boticão juntamente com a alavanca periapical, para a penetração entre dente e alvéolo, e assim foi concluída a extração do dente. Também foi necessária a utilização da grosa odontológica para corrigir muitos problemas de oclusão na dentição. Durante a cirurgia foram encontradas bolsas periodontais instaladas em alguns dentes repletas de restos de alimento (Figura 2).

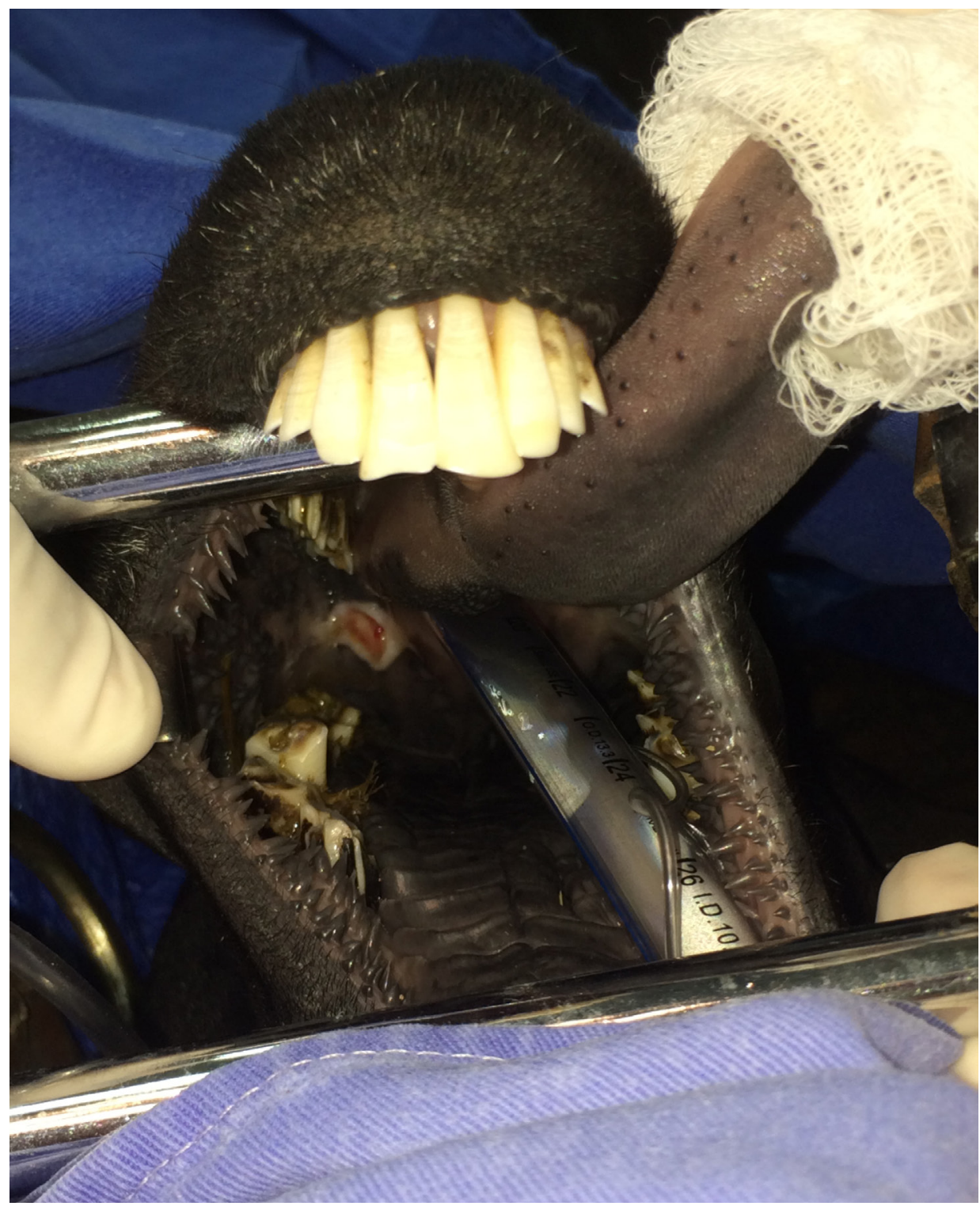

Figura 2 - Presença de restos de alimento em bolsas periodontais.

Fonte: Joyce C. R. Fioruci. 
Após a exodontia (Figura 3) a boca foi lavada com solução de $50 \mathrm{ml}$ de clorexidine alcoólico a $5 \%$. No local do dente extraído foi aplicado resina metilmetacrilato. No pós-operatório foi administrado flunixin meglumine intramuscular na dose de $1,1 \mathrm{mg} / \mathrm{kg}$, uma vez ao dia (SID), durante três dias; penicilina 6000000 UI na dose de 51.428 $\mathrm{UI} / \mathrm{kg}$ intramuscular, SID, durante cinco dias e gentamicina na dose de $2,5 \mathrm{mg} / \mathrm{kg}$ intramuscular, duas vezes ao dia (BID), durante três dias. Além disso, foi mantida a limpeza oral diária com a solução de clorexidina alcoólico a 5\%.

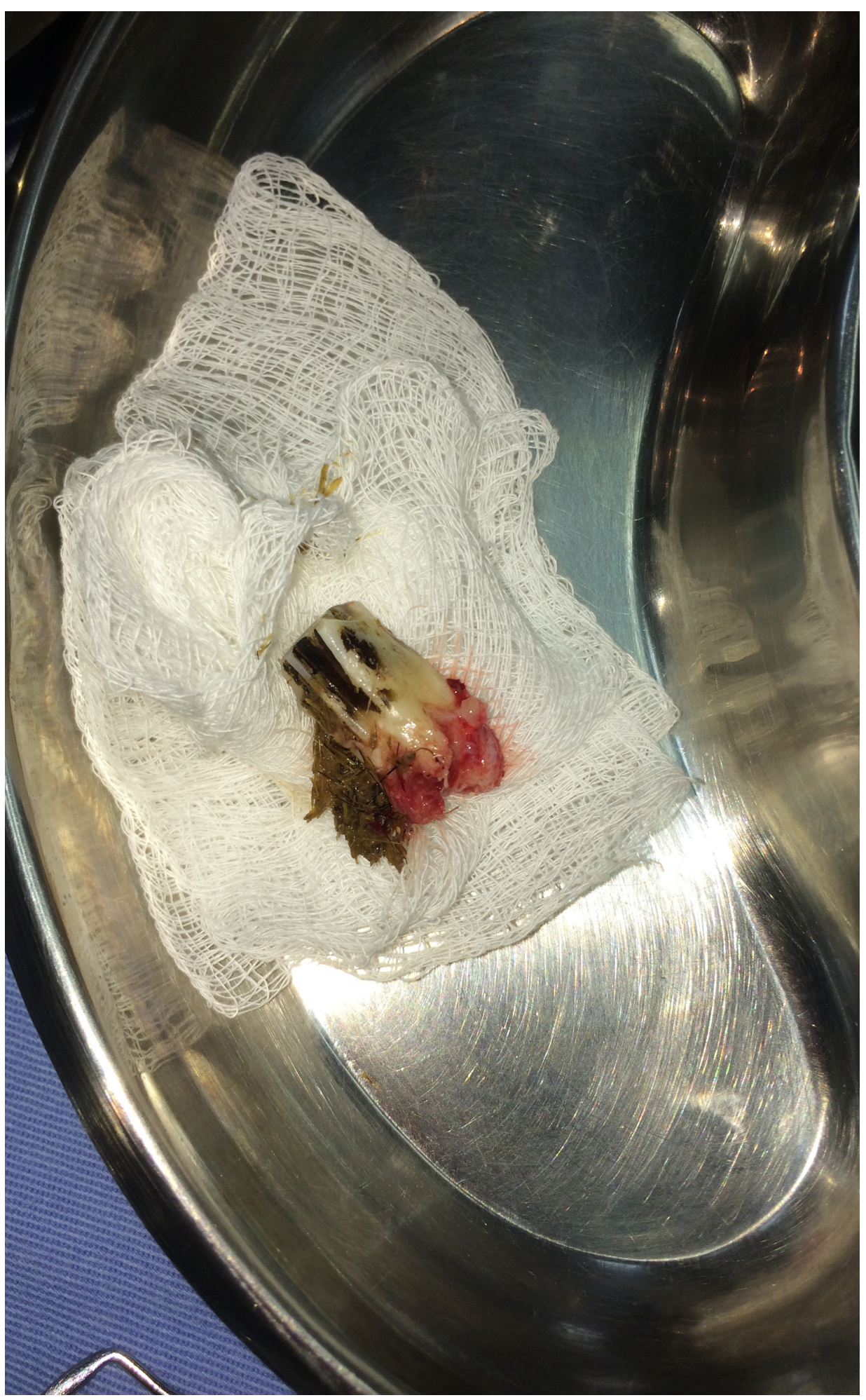

Figura 3 - Fotografia do $4^{\circ}$ pré-molar após a extração. Fonte: Joyce C. R. Fioruci. 


\section{Discussão}

O animal apresentou boa recuperação da cirurgia e em poucas horas já estava se alimentando e não aparentava dificuldades na mastigação. $\mathrm{O}$ atrito dentário anormal ocorre mais comumente em ovelhas do que em cabras. O pequeno ruminante adulto pode apresentar mais problemas relacionados à idade do que com doença dentária em si (PUGH, 2002).

A maior incidência de atrito dentário em ovinos ocorre em ovelhas de mais de quatro anos. A perda de dentes pode resultar em uma redução de dois anos na produtividade. Gnad et al. (2000) referem que a maioria das ovelhas e cabras pode acomodar a perda de um incisivo, mas a perda molar é mais grave, porque são dentes necessários para mastigação dos volumosos. Os sinais da existência de atrito dentário anormal incluem perda de peso, desgaste dentário, perda ou ausência de dentes. Os incisivos alongados podem resultar em mastigação incompleta ou dolorosa, trauma na almofada dentária ou tecidos moles, ou aparência anormal da região da face rostral (HUBER, 2004).

Com alta prevalência e por longos períodos presentes nos sistemas de produção de ovinos, os relatos registravam a baixa produtividade dos rebanhos, geralmente decorrentes do fato das fêmeas em reprodução parirem cordeiros menores e fracos, e ao abate precoce dos animais, que não expressavam plenamente seu potencial genético (WEST; SPENCE, 2000). Além de uma condição dolorosa, a afecção reduz a eficiência de pastejo do animal, contribuindo para má nutrição, perda de peso e problemas sistêmicos de saúde (ANDERSON; BULGIN, 1984; BAKER; BRITT, 1990).

\section{Considerações finais}

Concluiu-se que a cirurgia, neste caso, foi essencial para promover o conforto ao animal, para que assim pudesse voltar a se alimentar. Ressalta-se que ainda há necessidade de maior atenção para as enfermidades odontológicas nos pequenos ruminantes, visto que de acordo, estas enfermidades são responsáveis por grandes perdas econômicas em países como Nova Zelândia, Reino Unido e Austrália, pois interferem diretamente na produtividade do animal, uma vez que prejudica a sua mastigação e a ruminação, ocasionando emagrecimento, além de cordeiros nascidos mais leves, e descarte do animal antes mesmo do final de sua vida reprodutiva.

\section{Referências}

ANDERSON, B. C.; BULGIN, M. S. Starvation associated with dental disease in range ewes. Journal of the American Veterinary Medical Association, Schaumburg, v. 184, p. 737-738, 1984.
BAKER, J. R.; BRITT, D. P. Dental calculus and periodontal disease in sheep. Veterinary Record, London, v. 108, p. 331-333, 1990.

CUTRESS, T. W. et al. Periodontal disease in sheep. II. The composition of sera from sheep with periodontosis. Journal of Periodontology, Chicago, v. 43, p. 668-676, 1972.

GNAD, D. P. et al. Diagnosing weight loss in sheep: a practical approach. Compendium: continuing education for veterinarians, Yardley, v. 22, n. 1, p. 16-23, 2000.

GREENE, S. K. Equine dental advances. Veterinary Clinics North America Equine Practice, Philadelphia, v. 17, n. 2, p. 319-333, 2001.

HERRTAGE, M. E.; SAUNDERS, R. W.; TERLECKI, S. Physical examination of cull ewes at point of slaughter. Veterinary Record, London, v. 95, n. 12, p. 257-260, 1974.

HUBER, M. J. Dental surgery in small ruminants. of THE NORTH AMERICAN VETERINARY CONFERENCE, 131., 1994, San Francisco. Proceedings [...].Orlando: Journal of the American Veterinary Medical Association, 1994. p. 779-783.

JEFFERIES, B. C. Body condition scoring and its use in management. Tasmanian Journal Agricultural, Hobart, v. 32, p. 19-21, 1961.

KANE, D. M. The results of a Wairarapa survey of ovine incisor/ tooth anomalies with particular respect to wear. In: SEMINAR OF THE SOCIETY OF SHEEP AND BEEF CATTLE VETERINARIANS OF THE NEW ZEALAND VETERINARY ASSOCIATION, 14., 1984, Bulls. Proceedings [...]. Wellington: New Zealand Veterinary Association, 1984. p. 29-32.

ORR, M. B.; CHRISTIANSEN, K. H.; KISSLING, R. C. A survey of excessively worn incisors and periodontal disease in sheep in Dunedin city, Silverpeaks, Bruce and Clutha counties. New Zealand Veterinary Journal, Abingdon, v. 34, p. 111-115, 1986.

PUGH, D. G. Sheep goat medicine. Philadelphia: WB Saunders, 2002. $468 p$

PUGH, D. G. Clínica de ovinos e caprinos. São Paulo: Roca, 2004. $513 \mathrm{p}$.

SHERMAN, D. M. Unexplained weight loss in sheep and goats. a guide to differential diagnosis, therapy, and management. veterinary. Clinics North America: large animal practice, Philadelphia, v. 5, n. 3, p. 571-590, 1983.

WEINREB, M. M.; SHARAV, Y. Tooth development in sheep. American Journal of Veterinary Research, Schaumburg, v. 25, p. 891-908, 1964.

WEST, D. M.; SPENCE, J. A. Diseases of the oral cavity. In: MARTIN, W. B.; AIKEN, I. D. (ed.). Diseases of sheep. 3. ed. London: Blackwell Science, 2000. p. 125-131. 